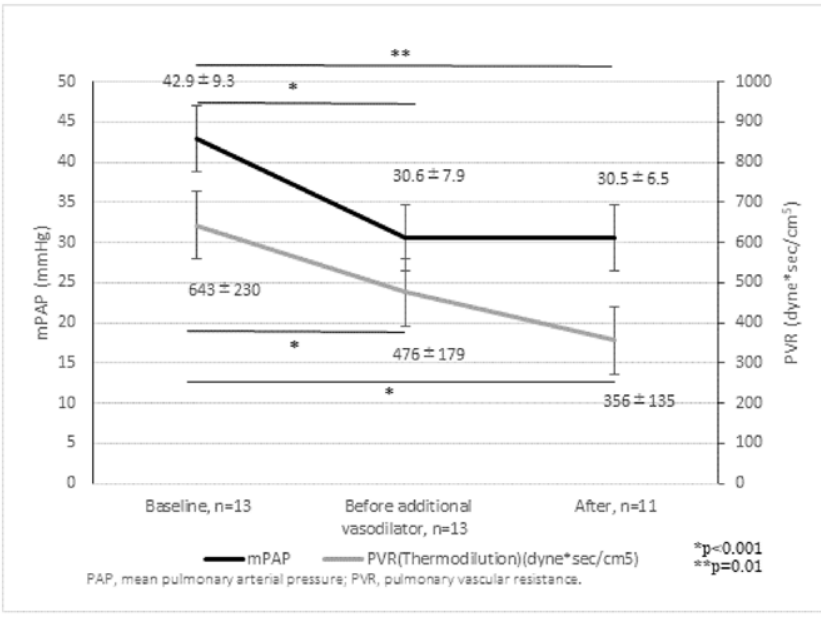

Figure 1. Hemodynamic changes during immunosuppressive therapy

Disclosure of Interests: Risa Kishikawa: None declared, Masaru Hatano Speakers bureau: Janssen Pharmaceutical K.K, Bayer Yakuhin, Ltd., Grant/ research support from: Janssen Pharmaceutical K.K, Nippon Shinyaku Co., Ltd., MOCHIDA PHARMACEUTICAL CO., LTD., Satoshi Ishii: None declared, Mai Shimbo: None declared, Akihito Saito: None declared, Shun Minatsuki: None declared, Yukiko Iwasaki: None declared, Keishi Fujio Speakers bureau: Tanabe Mitsubishi, Bristol Myers, Eli Lilly, Chugai, Jansen, Pfizer, Ono, AbbVie, Ayumi, Astellas, Sanofi, Novartis, Daiichi Sankyo, Eisai, Asahi Kasei, Japan Blood Products Organization, and Kowa, Grant/research support from: Tanabe Mitsubishi, Bristol Myers, Eli Lilly, Chugai, AbbVie, Ayumi, Astellas, Sanofi, Eisai, Tsumura \& Co., and Asahi Kasei., Issei Komuro Speakers bureau: AstraZeneka, Daiichi Sankyo Company, Limited, Takeda Pharmaceutical Company Limited, Bayer Yakuhin, Ltd, Pfizer Japan Inc., and Ono Pharmaceutical Co., Ltd., Grant/research support from: Daiichi Sankyo Company, Ltd, Sumitomo Dainippon Pharma Co., Ltd., Takeda Pharmaceutical Company Ltd, Mitsubishi Tanabe Pharma Corporation, Teijin Pharma Limited, Idorsia Pharmaceuticals Ltd, Otsuka Pharmaceutical Co., Ltd., Bayer Yakuhin, Ltd. Ono Pharmaceutical Co., Ltd. Toa Eiyo Ltd

DOI: 10.1136/annrheumdis-2021-eular.1573

\section{AB0286 EFFICACY OF MYCOPHENOLATE MOFETIL PLUS RITUXIMAB COMPARED WITH INTRAVENOUS CYCLOPHOSPHAMIDE PLUS RITUXIMAB IN PATIENTS WITH RELAPSING LUPUS NEPHRITIS}

L. D. Fajardo Hermosillo ${ }^{1}{ }^{1}$ Centro Médico Nacional de Occidente IMSS, Rheumatology, Guadalajara, Mexico

Background: Lupus nephritis (LN) remaining as one of the most devastating manifestations of systemic lupus erythematosus (SLE). Only $60 \%$ of patients with LN achieve a complete (CR) or partial remission (PR) with mycophenolate mofetil (MMF) or intravenous (iv) cyclophosphamide (CY) plus corticosteroids. Of those nearly one-half will have a relapse following maintenance therapy [1]. Rituximab (RTX) is considered a treatment of relapsing LN [2]. Although potential benefits have been revealed in descriptive studies, efficacy has not been established in randomized trials $[3,4]$.

Objectives: This study compares the efficacy achieving a CR or PR with $M M F+R T X$ versus iv $C Y+R T X$ in patients with relapsing $L N$.

Methods: Mexican SLE patients classified by SLICC 2012 criteria were recruited from 2013 to 2019 with LN diagnosed by renal biopsy who previously having achieved a CR with MMF or iv CY followed by the maintenance therapy with azathioprine (AZA) or MMF and subsequently present relapsing LN consisting of a new active urinary sediment (US), worsening proteinuria and renal function. Demographic, renal clinical and paraclinical variables were examined. All patients received oral prednisone $(1 \mathrm{mg} / \mathrm{kg} / \mathrm{d})$ accompanying MMF $3 \mathrm{~g} / \mathrm{d}$ or CY $0.5-1 \mathrm{~g} / \mathrm{m}^{2}$ monthly plus RTX $1 \mathrm{~g}$ at 0 and 14 days. The data were evaluated at $0,6,12$ and 24 month(s) after the start both treatments. CR was defined as normal serum creatinine (SCr), inactive US, plus 24 -Hour Urine Protein $(24 \mathrm{hUP})<500 \mathrm{mg} / \mathrm{d}$ and PC was established as 50 percent improvement in renal parameters. Fisher's exact and Student's-t tests were performed by univariate analysis.

Results: Of nine SLE patients with relapsing LN, seven were women. The mean age [standard deviation (SD)] was 25.1 (6.4) years. The mean time at the onset of SLE (SD) was 3.44 (1.12) years ago. Four and five patients had class IV and IV/V LN respectively. Time of relapsing LN after of CR (SD) was 21.2 (7.17) months. Seven patients used CY followed by AZA and two employed MMF previously of relapsing LN. The mean of basal SLEDAI and SLICC (SD) were 19.11(3.2) and 1.22 (0.44) respectively. The mean of basal 24hUP (SD) was 4.67 (2.6) g/d and sCr (SD) was $2.0(0.84) \mathrm{mg} / \mathrm{dl}$. Five patients used MMF+RTX and four patients utilized $C Y+R T X$. No statistically significant differences were found for CR, PR, 24hUP, sCr, US, anti-dsDNA and complement between both groups. At 6 months one patient achieved a CR and six reached a PR. At 12 months four patients fulfilled a CR and seven had a PR. Finally, at 24 months seven patients showed a CR and a CP. Only two patients did not achieve a CR or PR at 24 months.

Conclusion: This study suggests that the most of SLE patients with relapsing LN treated with CY or MMF plus RTX achieved CR or PR at 24 months, although were no found differences between both treatments. However, these observations must be confirmed in larger and prospective studies.

\section{REFERENCES:}

[1] Ann Rheum Dis 2020;79(6):713;

[2] Nephrol Dial Transplant 2019;34(1):22;

[3] Clin J Am Soc Nephrol 2009;4(3):579;

[4] Arthritis Rheum 2012;64(4):1215

Disclosure of Interests: None declared

DOI: 10.1136/annrheumdis-2021-eular.1612

\section{$\mathrm{AB} 0287$ \\ EFFECTS OF HYDROXYCHLOROQUINE ON PERIPHERAL BLOOD CYTOKINE EXPRESSION ASSOCIATED WITH ATHEROSCLEROSIS IN SYSTEMIC LUPUS ERYTHEMATOSUS}

R. Wakiya ${ }^{1}$, K. Ueeda ${ }^{1}$, H. Shimada ${ }^{1}$, S. Nakashima ${ }^{1}$, M. Kato ${ }^{1}$, M. Mahmoud Fahmy Mansour ${ }^{1}$, T. Miyagi ${ }^{1}$, K. Sugihara ${ }^{1}$, Y. Ushio ${ }^{1}$, M. Mizusaki ${ }^{1}$, R. Senba ${ }^{1}$, T. Kameda ${ }^{1}$, H. Dobashi ${ }^{1} .{ }^{1}$ Kagawa University, Division of Hematology, Rheumatology and Respiratory Medicine, Department of Internal Medicine, Kagawa, Japan

Background: In systemic lupus erythematosus(SLE), a higher frequency of atherosclerotic lesions is associated with a poor life prognosis (1). Hydroxychloroquine $(\mathrm{HCQ})$ has been reported to improve the prognosis of life and dyslipidemia in SLE (2), and the mechanism has been unclear.

Objectives: To determine the effect of HCQ treatment on serum cytokines associated with atherosclerosis in SLE.

Methods: SLE patients who received additional HCQ and maintained low dis ease activity between January 2016 and September 2020 were included in this study. Disease activity was assessed by SLEDAI, CLASI and LLDAS, and serum complement titers, anti-ds-DNA antibodies, serum insulin and serum cytokines (adiponectin, resistin and leptin) were analyzed before and after HCQ treatment.

Results: Fifty-four patients ( 3 males, 51 females, mean age $41.9 \pm 12.8$ years) were included (Table 1). Thirty-two patients achieved LLDAS at baseline. Serum adiponectin and insulin levels were significantly increased after 3 months of $\mathrm{HCQ}$ treatment compared to baseline, and serum resistin levels were significantly lower (Figure 1). Patients with a history of rena disease had greater degree of changes in serum adiponectin and resis tin levels. Among SLE patients who did not achieve LLDAS at baseline, those who still did not achieve LLDAS after 3 months had significantly lower serum leptin levels before HCQ treatment than those who achieved it after 3 months.

The change of serum resistin levels correlated with those of serum S100A8 levels $(r=0.5, p=0.0001)$

Conclusion: Additional HCQ treatment in SLE patients improves lipid abnormalities. HCQ may improve prognosis by controlling disease activity in SLE and reducing risk factors for atherosclerosis.

\section{REFERENCES:}

[1] Gregory Katz, et al. Systemic Lupus Erythematosus and Increased Prevalence of Atherosclerotic Cardiovascular Disease in Hospitalized Patients. Mayo Clin Proc. 2019; 94:1436-1443.

[2] Laura Durcan, et al. Longitudinal Evaluation of Lipoprotein Parameters in Systemic Lupus Erythematosus Reveals Adverse Changes with Disease Activity and Prednisone and More Favorable Profiles with Hydroxychloroquine Therapy. J Rheumatol. 2016; 43: 745-750. 